\title{
Imaging and Outcomes for a New Entity: Low-Grade Sinonasal Sarcoma with Neural and Myogenic Features
} \author{
Jason P. Hunt ${ }^{1,2}$ \\ ${ }^{1}$ Division of Otolaryngology - Head \& Neck Surgery, Department of \\ Surgery, University of Utah School of Medicine, Salt Lake City, \\ Utah, United States \\ 2 The Huntsman Cancer Institute, Salt Lake City, Utah, United States \\ ${ }^{3}$ Department of Radiology, University of Utah School of Medicine, \\ Salt Lake City, Utah, United States \\ ${ }^{4}$ Department of Pathology, University of Utah School of Medicine, \\ Salt Lake City, Utah, United States
}

Richard B. Cannon ${ }^{1,3}$ Richard H. Wiggins III, ${ }^{1}$ Benjamin L. Witt ${ }^{2,4} \quad$ Yusuf Dundar $^{2}$ Tawni M. Johnston ${ }^{2}$

\begin{abstract}
Address for correspondence Richard Cannon, MD, Division of Otolaryngology - Head \& Neck Surgery, Department of Surgery, University of Utah School of Medicine, 50 North Medical Dr., SOM 3C120, Salt Lake City, UT 84132, United States (e-mail: Richard.Cannon@hsc.utah.edu).
\end{abstract}

J Neurol Surg Rep 2017;78:e15-e19.

\author{
Abstract \\ Keywords \\ - low-grade sinonasal \\ sarcoma with neural \\ and myogenic \\ features \\ - skull base malignancy \\ - imaging \\ characteristics \\ - surgical outcomes \\ - hyperostotic bone \\ formation \\ - locally aggressive \\ - sinonasal sarcoma
}

Objectives Low-grade sinonasal sarcoma with neural and myogenic features (LGSSNMF) is a new, rare tumor. Our goal is to describe the imaging characteristics and surgical outcomes of this unique skull base malignancy.

Design Retrospective case series.

Setting Academic medical center.

Participants There were three patients who met inclusion criteria with a confirmed LGSSNMF.

Main Outcome Measures Imaging and histopathological characteristics, treatments, survival and recurrence outcomes, complications, morbidity, and mortality.

Results Patients presented with diplopia, facial discomfort, a supraorbital mass, and nasal obstruction. Magnetic resonance imaging and computed tomography imaging in all cases showed an enhancing sinonasal mass with associated hyperostotic bone formation that involved the frontal sinus, invaded the lamina papyracea and anterior skull base, and had intracranial extension. One patient underwent a purely endoscopic surgical resection and the second underwent a craniofacial resection, while the last is pending treatment. All patients recovered well, without morbidity or long-term complications, and are currently without evidence of disease (mean follow-up of 2.1 years). One patient recurred after 17 months and underwent a repeat endoscopic skull base and dural resection.

Conclusions The surgical outcomes and imaging of this unique, locally aggressive skull base tumor are characterized.

\section{Introduction}

Sarcomas of the head and neck are rare entities accounting for approximately $3 \%$ of head and neck malignancies. ${ }^{1}$ Sinonasal sarcomas are even rarer and create a diagnostic and treatment dilemma, particularly due to their varied tumor histologies. Review of the SEER (Surveillance, Epidemiology, and End Results) database showed that 5 -year survival was $47 \%$ for all sinonasal sarcomas, and increasing age, male sex, frontal and maxillary sinus subsites, and rhabdomyosarcoma and Kaposi sarcoma received

June 8, 2016

accepted after revision

December 21, 2016
DOI http://dx.doi.org/

10.1055/s-0037-1598198. ISSN 2193-6358. 〔c 2017 Georg Thieme Verlag KG
Stuttgart · New York

License terms

(c) $9 \ominus \$$ 
histologies were associated with a significant increase in mortality.

In 2012, a new tumor was described in this category, lowgrade sinonasal sarcoma with neural and myogenic features (LGSSNMF), with 28 cases review at the Mayo clinic and an additional case was reported in $2015 .^{2-5}$ This is a locally aggressive tumor with a propensity for recurrence. There is a 3:1 female-to-male ratio and age ranged from 24 to 85 years old (mean: 52 years). It is a rare head and neck malignancy with skeletal muscle differentiation that is not a rhabdomyosarcoma. ${ }^{6}$ All cases show a characteristic histology: uniform spindle cell neoplasm with elongate nuclei, neural and myogenic differentiation, and expression of S100 and most with actin positivity (93\%).

Most LGSSNMF involve the nasal cavity (55\%) or the ethmoids $(55 \%)$, with rare sphenoid $(7 \%)$ or frontal $(13 \%)$ involvement, and maxillary ( $0 \%$ ) involvement has not been described. Orbital and skull base involvement is not common ( $24 \%$ and $21 \%$, respectively) and only one case has demonstrated intracranial extension. Imaging characteristics have not been described for LGSSNMF. Follow-up was documented for $17 / 29$ (59\%), mean of 7.8 years, and $7 / 17$ (41\%) developed a recurrence and no patient has developed metastases or died of this disease. In contrast, sinonasal malignancies as a group have poor survival that depends on the histology with 5-year relative survival of 30 to $70 \%{ }^{7}$

This study aims to describe our surgical and oncological outcomes with this rare tumor, as well as the presentation and imaging features to further characterize this new skull base malignancy for the head and neck surgical oncologist, otolaryngologist, and neurologic surgeon.

\section{Case 1}

Our first patient is a 67-year-old female who presented with diplopia and facial discomfort. Computed tomography (CT) and magnetic resonance imaging (MRI) imaging identified a sinonasal mass in the right frontal recess with extension into the ethmoids, intimately associated with the lamina papyracea with hyperostotic bone formation ( - Fig. 1A, B). She underwent an endoscopic biopsy that confirmed a spindle cell neoplasm and also underwent an endoscopic anterior skull base resection with removal of the lamina papyracea. Surgical pathology showed a mucosal lesion comprising bland spindle cells without mitosis, necrosis, or pleomorphism, but the neoplastic proliferation was infiltrative into bone (-Fig. 2). This tumor stained focally positive for S100, smooth muscle actin (SMA), and desmin, and it was negative for CD34, Sox10, epithelial membrane antigen, and cytokeratin AE1/ AE3. It was evaluated by reverse transcription polymerase chain reaction for SS18 (SYT) gene translocation at location $18 q 11$, which was negative, and thus ruled out a synovial cell sarcoma. The final diagnosis was a LGSSNMF.

She recovered well, without morbidity, but recurred 17 months later with an avidly enhancing mass involving the anterior skull base and right medial rectus ( - Fig. 1C). She then underwent a revision endoscopic resection with removal of the adjacent anterior skull base and dura due to involvement demonstrated on the frozen section. Her intraoperative cerebrospinal fluid leak was repaired with a nasoseptal flap, and again she recovered well, without long-term morbidity.

\section{Case 2}

Our second patient is a 62-year-old female who presented with a slowly enlarging left supraorbital swelling. CT and MRI imaging showed a T1-enhancing, T2 hypointense mass in the left frontal sinus with hyperostotic bone formation that had eroded through the adjacent bone of the posterior table, contacted the dura, and pushed the frontal lobe posteriorly. It also extended inferiorly into the ethmoids, eroded the lamina papyracea, contacted the left medial rectus, and eroded the superior orbital wall (-Fig. 3). She underwent an endoscopic biopsy that showed LGSSNMF and then

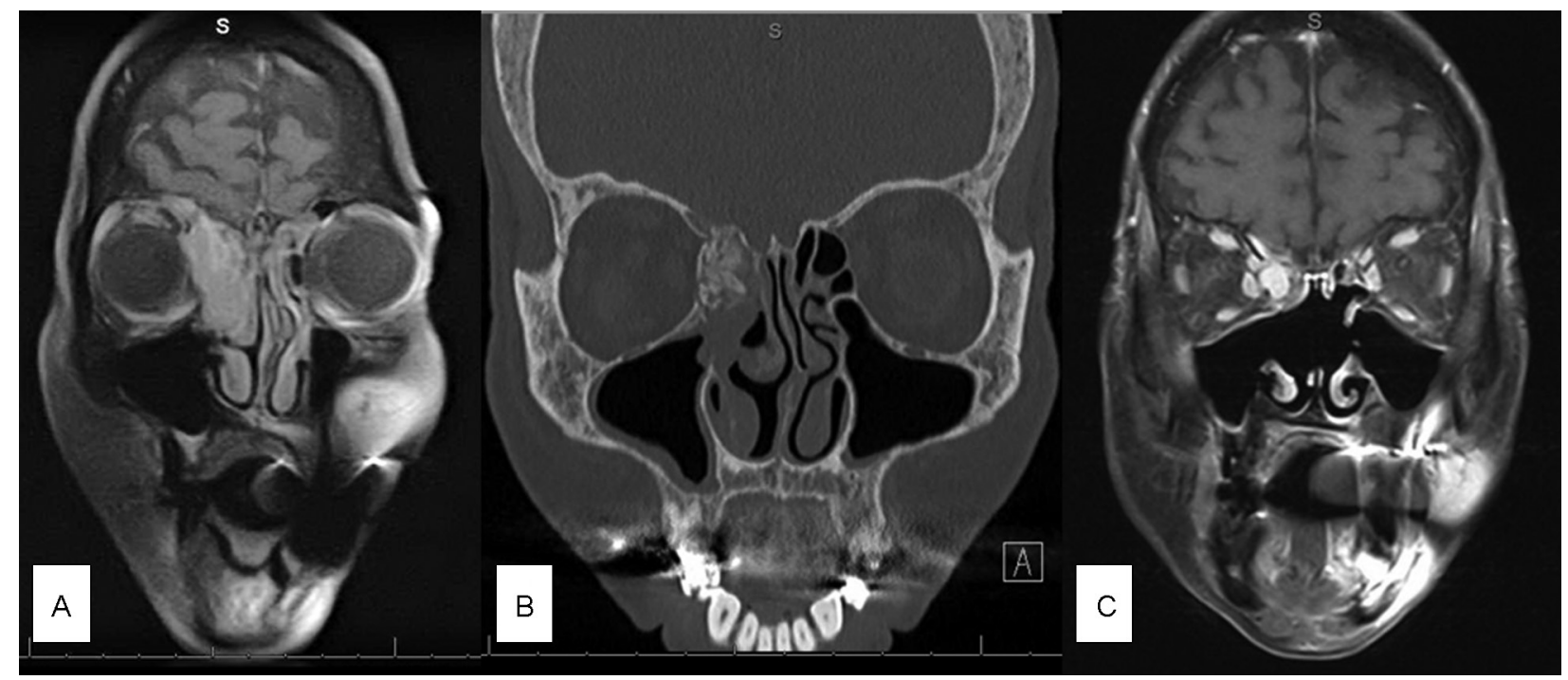

Fig. 1 Case 1. (A) Coronal T1 magnetic resonance imaging (MRI) and (B) coronal CT in bone window show the mass with hyperostotic bone formation with erosion through the adjacent skull base and lamina papyracea. (C) Coronal postcontrast fat-saturated T1 MRI shows the avidly, homogenously enhancing recurrent mass involving the skull base and right medial rectus muscle. 


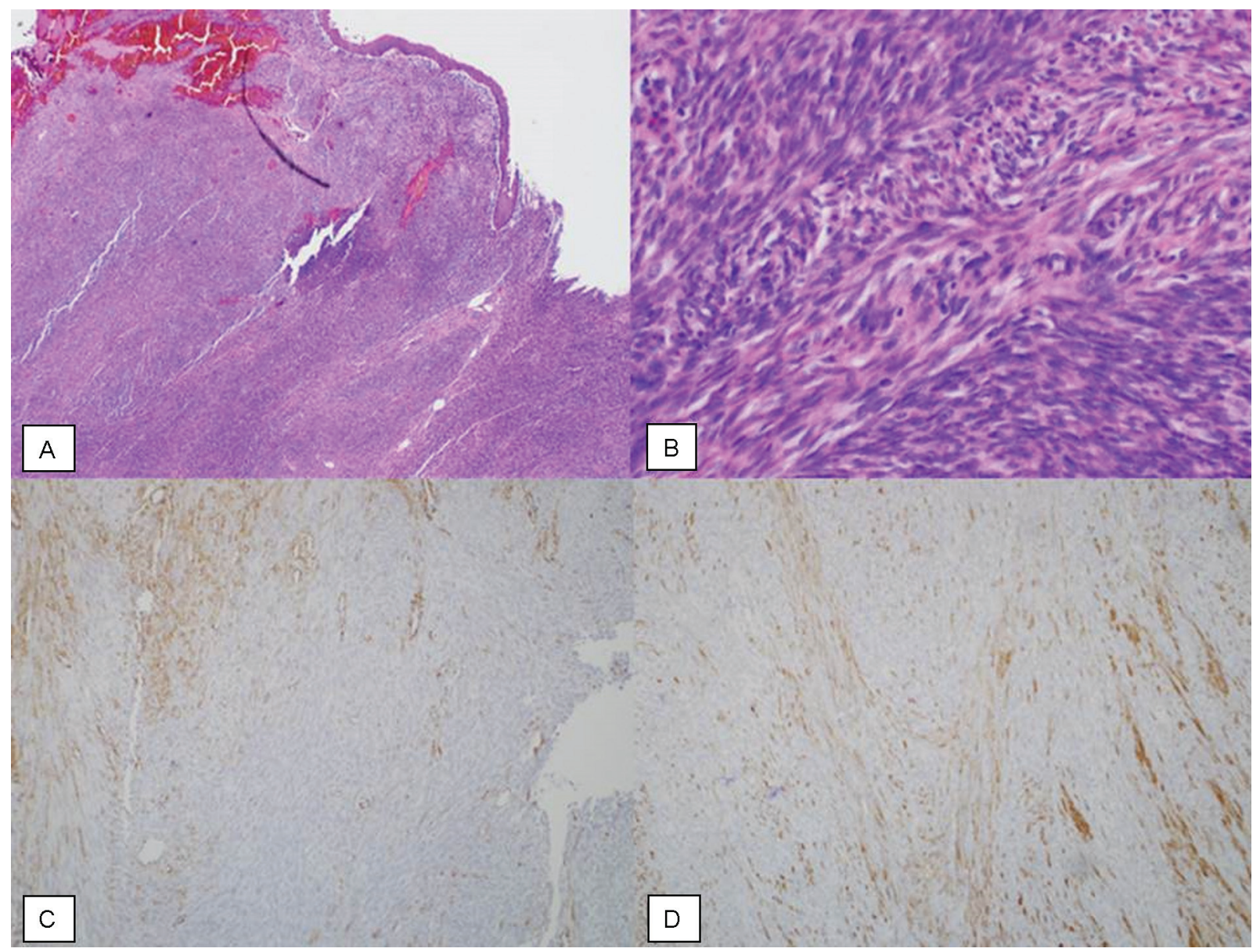

Fig. 2 Case 1. (A) Low-power $(4 \times)$ hematoxylin and eosin $(H \& E)$ stain shows the highly cellular lesion expanding the sinus submucosa. (B) Highpower $(40 \times) \mathrm{H} \&$ E stain shows the highly cellular but fairly bland and monotonous spindle cell neoplasm in a herringbone or fascicular pattern. (C) Intermediate power $(10 \times)$ shows patchy positive staining in the lesional cells for smooth muscle actin. (D) Intermediate power $(10 \times)$ shows patchy positive staining in the lesional cells for neural marker S100.

underwent a craniofacial resection of the anterior skull base in combination with neurosurgery. Final pathology showed a tumor focally eroding the bone comprising monotonous spindle cells without cytological atypia that were positive for S100 and SMA, and negative for desmin, myf4, and the SYT gene translocation. The final diagnosis was LGSSNMF, and she also recovered well, without morbidity.

\section{Case 3}

Our third patient is a 79-year-old female who presented with nasal obstruction and left facial pressure. CT and MRI imaging showed a T1-enhancing, T2 hypointense mass with hyperostotic bone formation of the left ethmoid sinuses that has eroded through the adjacent lamina papyracea and anterior

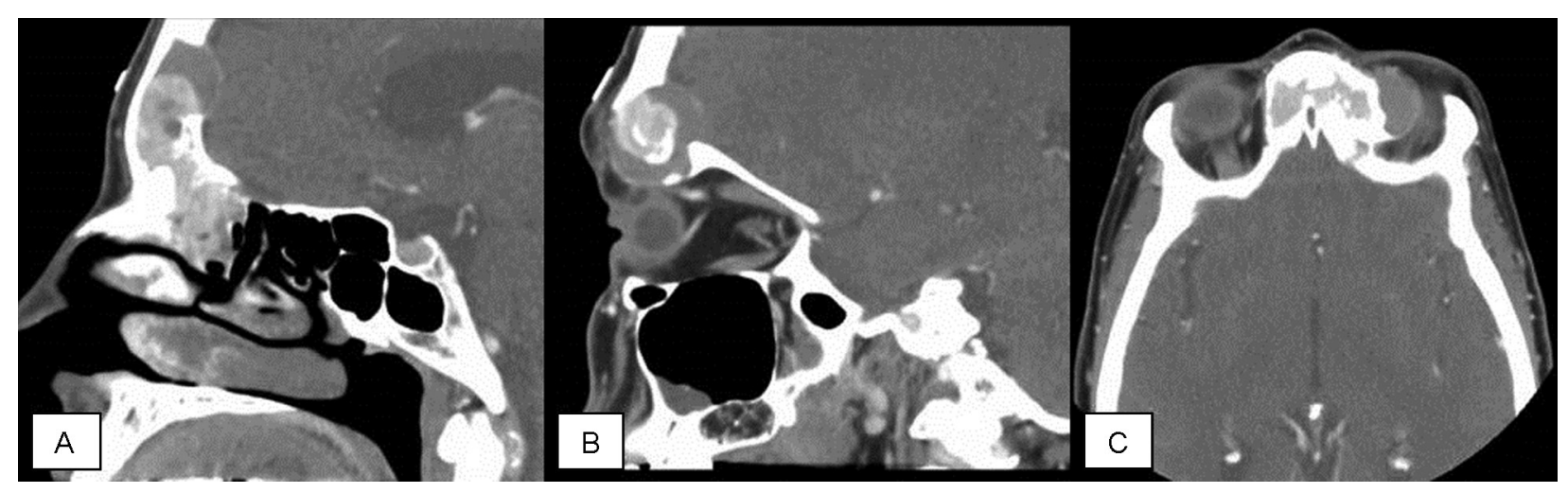

Fig. 3 Case 2. (A,B) Sagittal and (C) axial postcontrast computed tomography images show the frontal sinus mass with hyperostotic bone formation and erosion through the superior orbital wall into the orbit and through the posterior table of the skull base with intracranial extension. 


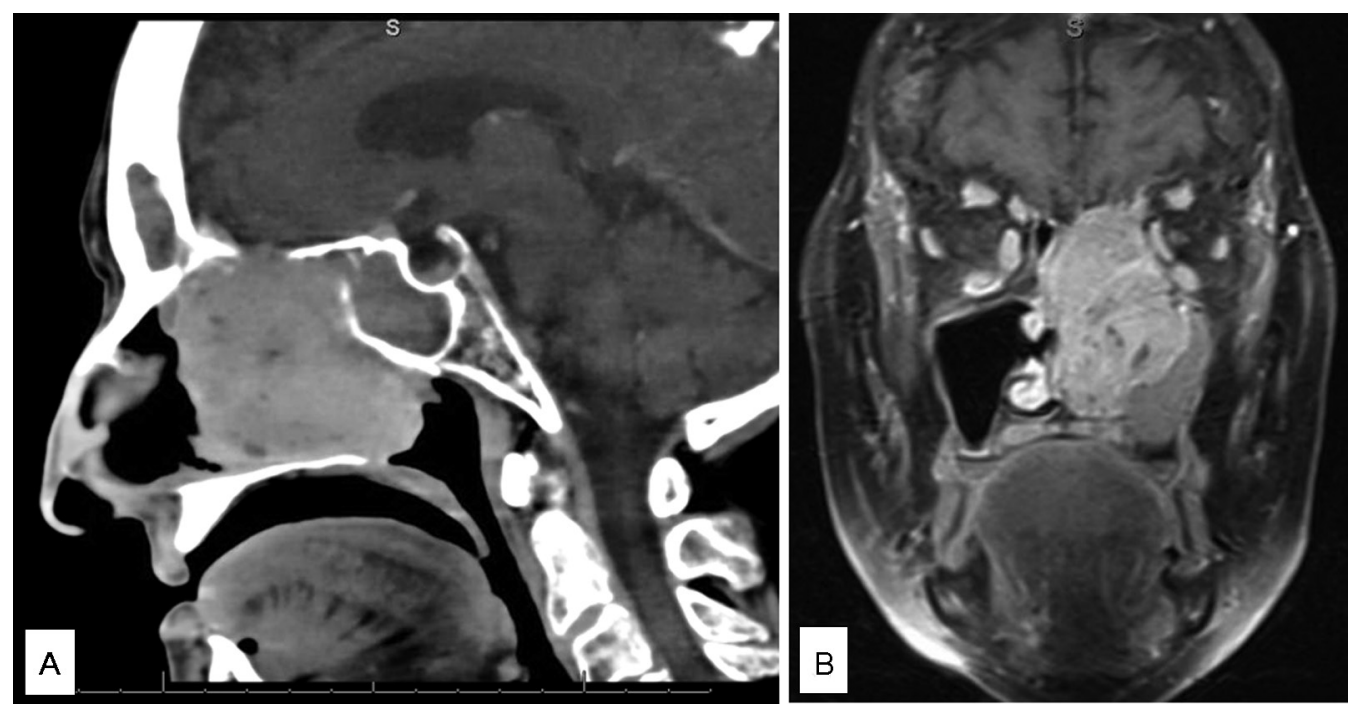

Fig. 4 Case 3. (A) Sagittal postcontrast computed tomography scan and (B) coronal postcontrast fat-saturated T1 magnetic resonance imaging images show the left sinonasal mass centered in the ethmoid cells with avid homogeneous enhancement and extension through the lamina papyracea and anterior skull base.

skull base and contacted the medial rectus and the dura (-Fig. 4). She underwent an endoscopic biopsy that showed LGSSNMF. It stained positive for S100, SMA, and desmin, and was negative for CD34, cytokeratin AE1/AE3, and muscle specific actin. SYT gene translocation was negative. She was found to have severe aortic stenosis in her work-up for surgery and underwent a transcatheter aortic valve replacement. She is recovering from this open chest surgery and still pending treatment for her skull base tumor.

\section{Discussion}

LGSSNMF is a very rare skull base tumor that is locally aggressive and has a propensity for recurrence with 29 prior cases reported. ${ }^{2,3}$ We illustrate three more cases and review the imaging characteristics of these tumors, which is lacking in the published literature. All our cases showed the characteristic histology with uniform spindle cell neoplasm with elongate nuclei, neural and myogenic differentiation, expres- sion of S100 and SMA, and negative SYT gene translocation. All tumors enhanced on MRI, had hyperostotic bone formation on CT scan, had eroded through the lamina papyracea, involved the frontal sinus, and had eroded through the anterior skull base with intracranial extension, which is rarely seen in the previously described cases.

Clinical features of the previously reported cases and our three patients are summarized in -Table 1. Our patients continue to highlight that this new, rare malignancy occurs predominantly in the elder (62-79 years old; average: 69 ), has a female predisposition, is infiltrative, particularly into bony structures, and usually involves the nasal cavity and ethmoids. Our patients all had a very locally aggressive variants with invasion into the lamina papyracea, anterior skull base, and dura, which was only described in 1 of the 29 previously published cases, and our patients had a high recurrence rate (50\%; mean follow-up of 2.1 years), which was similar to the previously published cases, and no patient has developed metastases or died of this disease.

Table 1 Clinical features of LGSSNMF in the published cases ${ }^{2,3}$ compared with our patients

\begin{tabular}{|l|l|l|}
\hline Clinical features & Published cases $(\boldsymbol{n}=\mathbf{2 9})$ & Our patients $(\boldsymbol{n}=3)$ \\
\hline Age & $24-85$ y old (average: 52$)$ & $62-79$ y old (average: 69) \\
\hline Male-to-female ratio & $1: 3$ & $0: 3$ \\
\hline Sinonasal involvement & $\begin{array}{l}\text { Nasal cavity (52\%), ethmoids (55\%), frontal } \\
(14 \%), \text { sphenoid }(7 \%), \text { maxillary (0\%) }\end{array}$ & $\begin{array}{l}\text { Nasal cavity (66\%), ethmoids (100\%), frontal } \\
(100 \%), \text { sphenoid (0\%), maxillary (0\%) }\end{array}$ \\
\hline Extension into the orbit & $7 / 29(24 \%)$ & $3 / 3(100 \%)$ \\
\hline Skull base involvement & $6 / 29(21 \%)$ & $3 / 3(100 \%)$ \\
\hline Intracranial extension & $1 / 29(3 \%)$ & $3 / 3(100 \%)$ \\
\hline Pathology & S100 (100\%), SMA (88\%), desmin (19\%), & $\begin{array}{l}\text { S100 (100\%), SMA (100\%), desmin (66\%), } \\
\text { CD34 (0\%), STY gene (0\%) }\end{array}$ \\
\hline Recurrent disease & $7 / 17(41 \%)$ in 7.8 y & $1 / 2(50 \%)$ in 2.1 y \\
\hline
\end{tabular}

Abbreviations: LGSSNMF, low-grade sinonasal sarcoma with neural and myogenic features; SMA, smooth muscle actin. 


\section{Conclusion}

LGSSNMF is a new, unique skull base malignancy, and the diagnosis, imaging, and treatment are reviewed. All tumors enhanced, had hyperostotic bone formation, and were locally aggressive with erosion through the lamina papyracea and anterior skull base, with extension into the dura, which is rare in the previously described cases. This tumor is important to know for those operating on the skull base.

\section{Support/Funding}

No sources of support or funding were received for this work.

\section{Conflict of Interest}

None of the authors has a conflict of interest.

Note

Presented at the 2016 Combined Sections Meeting, January 22 to 24, Miami Beach, Florida, United States.

\section{References}

1 Wu AW, Suh JD, Metson R, Wang MB. Prognostic factors in sinonasal sarcomas: analysis of the surveillance, epidemiology and end result database. Laryngoscope 2012;122(10): 2137-2142

2 Lewis JT, Oliveira AM, Nascimento AG, et al. Low-grade sinonasal sarcoma with neural and myogenic features: a clinicopathologic analysis of 28 cases. Am J Surg Pathol 2012;36(04):517-525

3 Powers KA, Han LM, Chiu AG, Aly FZ. Low-grade sinonasal sarcoma with neural and myogenic features-diagnostic challenge and pathogenic insight. Oral Surg Oral Med Oral Pathol Oral Radiol 2015;119(05):e265-e269

4 Riddle NN, Gardner JM. The new kids on the block: recently characterized soft tissue tumors. Surg Pathol Clin 2015;8(03): 467-491

5 Wenig BM. Recently described sinonasal tract lesions/neoplasms: considerations for the new world health organization book. Head Neck Pathol 2014;8(01):33-41

6 Bishop JA, Thompson LD, Cardesa A, et al. Rhabdomyoblastic differentiation in head and neck malignancies other than rhabdomyosarcoma. Head Neck Pathol 2015;9(04):507-518

7 Turner JH, Reh DD. Incidence and survival in patients with sinonasal cancer: a historical analysis of population-based data. Head Neck 2012;34(06):877-885 